\title{
The Effect of L1-L2 Lexicalization Mismatch on Incidental Acquisition of Receptive Vocabulary Knowledge
}

\author{
Mohammad Ali Heidari-Shahreza \\ Department of English, Faculty of Humanities, Shahreza Branch, Islamic Azad University, \\ Shahreza, Iran \\ E-mail address: maheidari.sh@gmail.com
}

\begin{abstract}
The researcher was interested to explore the possible effects of L1-L2 lexicalization mismatch on the acquisition and retention of receptive vocabulary knowledge by 90 Persian-speaking EFL learners. Lexicalization mismatch was defined in the context of this study as the lack of a lexically equivalent translation in learners' L1 (i.e. Persian) for an L2 word (i.e. English). Thus, non-lexicalized words referred to those L2 words that required a longer string of L1 words to cover their essential semantic features. The findings indicated that there were significant differences between lexicalized and nonlexicalized target words in the receptive knowledge of meaning and form and receptive knowledge of associations. Thus, it seems non-lexicalized words are most likely to cause extra difficulty for EFL learners in the semantic aspects of vocabulary knowledge. Using enhanced input within a systematic vocabulary recycling program is recommended to deal with such words.
\end{abstract}

Keywords: Lexicalization mismatch; Incidental vocabulary acquisition; L1 translation; Persianspeaking EFL learners

\section{INTRODUCTION}

The literature clearly suggests that L1 exerts significant influence on L2 vocabulary acquisition and use (Harding, 2011; Heidari-Shahreza (d-f), Moinzadeh, Barati, 2014 a-c). As for learners' output, research shows that quite a number of EFL learners' errors can be traced back to L1 influence (Hemchua, \& Schmitt, 2006). L1-L2 translation is also among the popular strategies used by learners to comprehend reading passages. It is also practiced as an effective technique by many language teachers in EFL contexts (Tagashira, Kida \& Hoshino, 2010). Yet, probably, the best evidence to show the significance of L1 knowledge comes from psycholinguistic studies, which suggest that learners' L1-oriented mental lexicon plays an important role during L2 lexical processing even in advanced learners (e.g. Sunderman \& Kroll, 2006). In short, the effects of L1 on a notable range of topics such as morphological awareness, cognate recognition, syntactic and semantic transfer have been explored (see for example, Harding, 2011; Heidari-Shahreza, 2014). Nevertheless, as Paribakht (2005) warns further research is required to account for possible effects of L1 lexical knowledge on L2 lexical 
processing and vocabulary acquisition. In this regard, one neglected area of problem for EFL learners is the issue of L1-L2 lexicalization mismatch.

\section{1. Lexicalization Mismatch}

Lexicalization refers to how a language forms different concepts into words or lexical items (Heidari-Shahreza \& Tavakoli, 2012; Paribakht, 2005). The point here is languages may have different ways of lexicalizing (or coining) the same concept (Paribakht, 2005; Chen and Truscott, 2010). L1 lexicalization indicates the way learners' first language (L1) lexicalizes different concepts that might be different from that of their second or foreign language (Chen \&and Truscott, 2010). In this regard, L1-L2 lexicalization mismatch addresses the question of whether or not L2 target words have the same translation in learners' L1 (e.g. Persian). Therefore, the L2 words which have a lexically equivalent translation are called 'lexicalized' while those words that need to be translated with a long string of words to cover their essential semantic components are named 'non-lexicalized' (Chen \& Truscott, 2010; Heidari-Shahreza, \& Tavakoli, 2012; Heidari-Shahreza et al., 2014 b). The word 'bacon' in English, for example, does not have an equivalent translation in Persian and has to be paraphrased in several words as 'كوشت باريكه شده و نمك زده يهلو يا بشت خوك ' Therefore, for Persian-speaking EFL learners, the word 'bacon' is considered a non-lexicalized (NL, hereafter) word. Whereas the word 'fish', for example, is easily lexicalized into one single Persian word, 'ماهى'. Hence, it falls into the category of lexicalized (L) English words with respect to Persian.

\section{LITERATURE REVIEW}

Among the few studies with a special focus on L1-L2 lexicalization mismatch is Paribakht's seminal study (2005) in which she investigated the relationship between L1-L2 (i.e. Persian and English) lexicalization process and the inferencing behavior of 20 Persian-speaking learners while reading English passages. The findings revealed that while the participants made use of the same knowledge resources in inferencing both L and NL words, they had more difficulty in decoding NL words. Paribakht concluded that L1-L2 lexicalization mismatch might be to the detriment of learners' L2 reading comprehension and vocabulary development. Her study, however, did not explore how L and NL words were different in the acquisition and retention of different aspects of vocabulary knowledge.

To bridge this gap, Chen and Truscott (2010) using a modified version of Webb's taxonomy of vocabulary knowledge (2007), explored the incidental vocabulary acquisition and retention of 10 target words by 72 university students. They found that NL words could cause learning difficulty both immediately and after two weeks. It was further suggested that even an increase in exposure frequency up to seven encounters could contribute little to significant learning of NL words since, in their view, these words were "too difficult to learn form even seven encounters ". Their study, however, was limited in that, among other things, the position of the target words in the reading passages, their saliency and informativeness were not fully controlled.

Based on this study, Heidari-Shahreza and Tavakoli (2012) further investigated the same issue on 90 Iranian EFL learners using 10 English target words. They concluded that NL words caused learning difficulty mainly in semantic aspects of vocabulary knowledge such as knowledge of meaning and associations. However, despite some improvements over Chen and 
Truscott's study, their study did not control for the potential cultural connotations of the target words.

\section{THE STUDY}

As the small number of studies on L1 Lexicalization in relation to vocabulary acquisition indicates, further research on this factor is needed. Hence, this study, through a quasiexperimental design investigated the possible effects of L1-L2 lexicalization mismatch on successful incidental vocabulary acquisition and retention by 90 Persian-speaking EFL learners. Furthermore, the researchers were interested to know how any observed gains in learners' vocabulary knowledge are retained over a period of three weeks. Thus, retention is also taken into account by a delayed posttest. This study is part of a much larger project exploring, number of exposure frequency, L1 lexicalization, L2 equivalence and cultural loadedness, to be reported.

The present study embarked on answering three research questions:

1. Did the acquisition of non-lexicalized target words differ from the ones that were lexicalized in EFL learners' L1 (i.e. Persian)?

2. If so, which aspects of receptive vocabulary knowledge were more involved?

3. Was there any significant difference between lexicalized and non-lexicalized target words in terms of retention after three weeks?

\section{1. Participants}

The population, out of whom the final participants were selected, was an Iranian University. A call on voluntary participation was announced and 128 students expressed their interest to participate. They, then, took Oxford Placement Test out of which 111 were identified as intermediate. Afterwards, the Vocabulary Levels Test (Nation, 1990), a widely-used size test and an appropriate measurement instrument for vocabulary knowledge was given. All participants scored 25 or more (out of 30) on 2000 level of the Vocabulary Level Test, with an average score of 28.2.

As the third stage, the participants filled a sociolinguistic background survey through which it was assured that the final participants, among other things, had the same first language and amount of exposure to English. Due to a significantly different performance on the Levels test or their linguistic background, a few participants were excluded from the scope of this study. Finally, 90 participants were deemed as sufficiently appropriate for this study. They were then, equally divided into three groups of participants, based on the number of encounters to TWs (i.e. E1, E3, and E7).

\section{2. Materials and Instruments}

\section{2. 1. Target words}

There were 10 target words (TWs, hereafter) which were equally classified into two groups:

- Lexicalized (L);

- Non-lexicalized (NL); 
They all together included four verbs, four nouns and two adjectives (see Table 1). To select the TWs, the researchers decided to prepare a small corpus of lexicalized and nonlexicalized words. In so doing, after a call on participation, 40 university students who were native speakers of Persian and fluent in English volunteered to note down the appropriate words they encountered over a six-month period of reading English texts. Before embarking on that, the researchers held several meetings with them, to define and provide examples for $\mathrm{L}$ and NL words. Over these 6 months, three more meetings were held to make sure they were all on the right track. The assistants were also kindly asked to check their initial guess regarding the suitability of a word against well-established English as well as Persian dictionaries and ask other native speakers, if necessary. All collected words were also accompanied by one example of its use in an authentic context. As the outcome of this cooperation, more than 1500 words were gathered (mainly verbs, nouns, adjectives and few adverbs). The researchers checked these words once more that resulted in excluding a few ones. Afterwards, based on the difficulty level, conceptual familiarity and word frequency, 10 words were deemed as final TWs. It is worth noting that selected TWs were assured to be unknown to all the participants at the time of the study, based on a checklist.

Table 1. Selected TWs.

\begin{tabular}{cc}
\hline Lexicalized (L) & Non-lexicalized (NL) \\
\hline explain (v) & elope (v) \\
flee (v) & giggle (v) \\
annoyance (n) & lounge (n) \\
masterpiece (n) & brunch (n) \\
stubborn (adj) & smoggy (adj) \\
\hline
\end{tabular}

\section{2. 2. Reading passages}

On the whole, the participants read 13 reading passages. These passages were of two types: Main reading passages (M) which each contained all 10 TWs once and distracter passages (D) which despite the same length (more or less 250 words) and difficulty level, did not contain any of TWs. Based on the design of the study (i.e. one, three or seven encounters to TWs), seven main passages were composed by the researchers and two native English teachers. The other six remaining distracter passages were taken from a reading textbook at intermediate level (Kirn \& Hartmann, 2002) only for the participants to read the same number of reading passages regardless of which experimental group (i.e. E1, E3 or E7) they were in (see Table 2).

Table 2. Distribution of reading passages.

\begin{tabular}{ccccccccc}
\hline Group & \multicolumn{7}{c}{ Distribution of Main and Distracter passages } & exposure \\
\hline E1 & $\mathrm{D}_{1}$ & $\mathrm{D}_{2}$ & $\mathrm{D}_{3}$ & $\mathrm{D}_{4}$ & $\mathrm{D}_{5}$ & $\mathrm{D}_{6}$ & $\mathrm{M}_{7}$ & $\mathbf{1}$ \\
E3 & $\mathrm{M}_{1}$ & $\mathrm{D}_{2}$ & $\mathrm{D}_{3}$ & $\mathrm{M}_{4}$ & $\mathrm{D}_{5}$ & $\mathrm{D}_{6}$ & $\mathrm{M}_{7}$ & $\mathbf{3}$ \\
E7 & $\mathrm{M}_{1}$ & $\mathrm{M}_{2}$ & $\mathrm{M}_{3}$ & $\mathrm{M}_{4}$ & $\mathrm{M}_{5}$ & $\mathrm{M}_{6}$ & $\mathrm{M}_{7}$ & $\mathbf{7}$ \\
\hline
\end{tabular}




\section{2. 3. Vocabulary post-test}

To have a better picture of the learners' vocabulary knowledge after exposing to TWs, as in Chen and Truscott's (2010) study, a modified version of Webb's (2007) test of vocabulary knowledge was used. The multifaceted focus of this test allowed for assessing different aspects of receptive vocabulary knowledge (see Table 3).

Table 3. Types of vocabulary knowledge \& vocabulary post-test sub-tests.

\begin{tabular}{ccc}
\hline No. & Knowledge measured & Test type \\
\hline $\mathbf{1}$ & Receptive Knowledge of Orthographic Form (RO) & Multiple choice \\
$\mathbf{2}$ & Receptive Knowledge of Meaning and Form (RMF) & Translation (L2-L1) \\
$\mathbf{3}$ & Receptive Knowledge of Parts of Speech (RP) & Multiple choice \\
$\mathbf{4}$ & Receptive Knowledge of Associations (RA) & Multiple choice \\
\hline
\end{tabular}

\section{Sub-test 1. Receptive Knowledge of Orthographic Form (RO)}

To test the receptive knowledge of orthographic form, a four-option multiple choice format was employed. Distracters in each test item resembled the TW in spelling. Below is an example for the TW, 'stubborn':
a. stobborn
b. stubborn
c. stubbern
d. stuborn

\section{Sub-test 2. Receptive Knowledge of Meaning and Form (RMF)}

To test this aspect of knowledge, the researchers asked the participants to translate the TWs into their L1 (i.e. Persian). Participants scored one point if all essential semantic components of a TW were realized in their translations. For example, for the TW, 'elope', an answer such as 'run' was considered as wrong since the semantic feature [+ secret] was not included.

\section{Sub-test 3. Receptive Knowledge of Parts of Speech (RP)}

To successfully pass this subtest, the participants need to know which grammatical functions the TWs could have. To measure this knowledge, three (almost) similar sentences were given in which the TWs had different grammatical functions. The participants were told to check the sentence in which the TW had the correct grammatical function, as shown in the following example for the TW, 'explain' as a verb:

(a) She made up an explain about the situation.

(b) She explained the situation to her father ${ }^{\checkmark}$

(c) Her explain idea made the situation worse.

\section{Sub-test 4. Receptive Knowledge of Associations (RA)}

In this test, each TW came with five words. The researchers instructed the test-takers to identify those words that were pragmatically associated with the TWs. The advantage of 
allowing more than one correct answer for each test item was that it revealed whether the learners knew all essential semantic features of NL words or not (as in Chen, \& Truscott, (2010) and Heidari-Shahreza, \& Tavakoli, (2012). Below, The TW, 'giggle' and its five options are presented in which (c) and (e) are expected answers:
(a) talk
(b) noisy
(c) laugh ${ }^{2}$
(d) shake
(e) silly

The researchers piloted the subtests with a similar group of participants. Several test distracters were replaced with more appropriate options and instructions were made clearer especially for the subtest seven in which the participants needed to choose more than one correct answer. The average reliability was calculated as 0.84 .

\section{3. Data Collection}

As mentioned above, there were 13 reading passages (seven main and six distracter texts). However, each group was to read only seven passages. Based on the design of the study, the first six reading passages in group E1were distracters. Therefore, they only read one main passage containing the TWs. Group E3 read three main and four distracter passages hence they had three encounters to the TWs. Unlike E1 and E3, group E7 did not read any of the distracters. That is, they read all seven main reading passages. Therefore, they had seven encounters to the TWs. It is worth noting that the seventh passage in all groups was a main passage and thus contained the TWs. Being so, all the participants had finished the reading phase an encounter to the TWs. This, in turn, blocked the effect of how recently they had seen the TWs.

Upon having read the passages, the participants sat for the vocabulary post-test outlined above. Although the participants did not expect a vocabulary post-test, they were willing enough to take it. Each subtest was printed on a single page and the participants were told not to look back to the preceding subtests. There was no time limit on the submission of the answer sheets. Yet, the test-takers finished the test within an acceptable time range. To check participants' retention of any gained vocabulary knowledge from reading the passages, the participants again, take the vocabulary post-test after three weeks. There was no sample attrition and the test proceeded following the same procedure as the immediate post-test. In addition, as far as feasible, the participants' exposure to English usually via learning tasks or reading materials were generally considered by the researchers during these three weeks to control for any significant effect on their vocabulary knowledge.

\section{4. Data Analysis}

To analyze the scores obtained from the participants in the three experimental groups (i.e. E1, E3 and E7) ANOVA and its non-parametric version Kruskal-Wallis were employed (whenever normality requirement was not met). Moreover, Post hoc Tukey and least significance difference (LSD) tests were used to discern significant effects (at $p=.05$ ). The same statistical procedure was run for the results obtained from the delayed posttest. In the next section, the results are presented in details.

\section{RESULTS}

\section{1. Effects of L1 Lexicalization in the Immediate Post-test}

Based on the analyses of mean score differences between the groups, the participants in all groups did significantly better for L words than NL words in the Receptive Knowledge of 
Associations (RA) test. The Receptive Knowledge of Meaning and Form (RMF) was also another aspect of vocabulary knowledge where the scores for $\mathrm{L}$ words were significantly higher than NL words for groups E3 and E7 (see Table 4).

Table 4. Comparison between $\mathrm{L}$ and NL words in the immediate post-test.

\begin{tabular}{cccc}
\hline Group & E1 & E3 & E7 \\
\hline Sub-test & L vs. NL & L vs. NL & L vs. NL \\
Receptive Knowledge of Orthographic Form & 0.632 & 0.487 & 0.584 \\
Receptive Knowledge of Meaning and Form & 0.246 & $0.003^{*}$ & $0.024^{*}$ \\
Receptive Knowledge of Parts of Speech & 0.734 & 0.354 & 0.386 \\
Receptive Knowledge of Associations & 0.254 & $0.023^{*}$ & $0.005^{*}$ \\
\hline
\end{tabular}

Note: $*=p<.05$; L: Lexicalized; NL: Non-lexicalized

\section{2. Effects of L1 Lexicalization in the Delayed Post-test}

Based on the same statistical procedure, the mean scores for L and NL words were analyzed to discern how a three-week delay could make a difference in the observed results for the immediate post-test. As shown in Table 5, the same significant differences were observed again in the delayed vocabulary post-test.

Table 5. Comparison between L and NL Words in the Delayed Post-test.

\begin{tabular}{cccc}
\hline Group & E1 & E3 & E7 \\
\hline Sub-test & L vs. NL & L vs. NL & L vs. NL \\
Receptive Knowledge of Orthographic Form & 0.557 & 0.499 & 0.798 \\
Receptive Knowledge of Meaning and Form & 0.288 & $0.020^{*}$ & $0.001^{*}$ \\
Receptive Knowledge of Parts of Speech & 0.456 & 0.564 & 0.704 \\
Receptive Knowledge of Associations & 0.062 & $0.006^{*}$ & $0.021^{*}$ \\
\hline
\end{tabular}

Note: $*=p<.05$; L: Lexicalized; NL: Non-lexicalized

\section{DISCUSSION}

The primary purpose of the present study was to investigate the possible effects of L1-L2 lexicalization mismatch on the acquisition and retention of receptive knowledge of vocabulary. Simply put, the study aimed at discerning how the acquisition and retention of non-lexicalized (NL) words would differ from lexicalized (L) words with respect to different aspects of receptive vocabulary knowledge. A fundamental question of this study was whether or not NL words could possibly cause learning difficulty for EFL learners. In this regard, the findings of the study generally indicates that the main difference between L and NL words lies in the semantic aspects of vocabulary knowledge (as also concluded by Heidari-Shahreza and 
Tavakoli, 2012; Heidari-Shahreza et al., 2014 b,c). That is to say, there were significant differences in the mean scores obtained by the participants for NL words in comparison with their L counterparts on the semantic subtests of receptive knowledge of meaning and form (RMF) and receptive knowledge of associations (RA) in the immediate posttest after seven encounters (i.e. E7). A complication to this general pattern of the semantic tests is that while even after three encounters, significant differences between $\mathrm{L}$ and NL words were observed on RMF and RA tests, the mean score differences between the two sets of vocabulary reached significance only after seven encounters on PA test.

Another important question here is why significant differences were observed on the semantic aspects of vocabulary knowledge (i.e. meaning and association) and not the syntactic and orthographic ones. While certainly further research is needed, it might be due to the active role of L1 in L2 lexical inferencing and meaning construction. The literature in this regard, suggests that the initial form-meaning linkage of an L2 word is mediated by the learners' L1 lexicon (Barcroft, 2002). That is, a new L2 form is initially attached to an already existing meaning in the learners' L1-based mental lexicon. During the process of lexical inferencing from a text, cognitively speaking, EFL learners seek for the best match in their mental lexicon for the new L2 word, based on the cues extracted from the context (see Barcroft, 2004). As for an L word, the lexical equivalent is readily retrieved from a learner's L1-based lexicon since it is already existing as a 'lemma package' (as Paribakht, 2005 calls it). However, the process of lexical matching (or L1-L2 mental translation, so to speak) is deterred for an NL word because there is no existing or largely overlapping lemma (i.e. an appropriate match) for it in the mental lexicon. Therefore, given that EFL learners can extract the semantic features of an NL word from the surrounding text, they may not be able to fully acquire the meaning of that word since an NL word cannot trigger a corresponding lemma in the mental lexicon (Heidari-Shahreza \& Tavakoli, 2012; Paribakht, 2005). Therefore, it seems plausible why the participants were particularly less successful in the semantic aspects of vocabulary knowledge in acquiring NL words.

\section{CONCLUSIONS}

As the primary aim of this study, the researchers were particularly interested to explore the possible effects of L1-L2 lexicalization mismatch on the acquisition and retention of vocabulary knowledge. Lexicalization mismatch was defined in the context of this study with respect to two sets of the target words (TWs): lexicalized (L) and non-lexicalized (NL) words. L words, in essence, represented a large number of English words that could be easily translated to (or replaced with) their equivalents in the learners' L1 (here, Persian) with the same number of lexical items. NL words which were in fact a marked portion of L2 vocabulary, referred to those L2 words that required a longer string of L1 words to cover their essential semantic features. Based on this definition, the study focused on the acquisition and retention of $10 \mathrm{TWs}$ (including $\mathrm{L}$ and NL words) through reading English texts by 90 Iranian adult EFL learners. The findings generally indicated that there were significant differences between L and NL words in the semantic aspects of vocabulary knowledge such as receptive knowledge of meaning and form (RMF) and receptive knowledge of associations (RA). As for the other aspects of vocabulary knowledge such as receptive and productive knowledge of orthography or parts of speech, this study did not bear any significant results.

Overall, it seems NL words are most likely to cause extra difficulty for EFL learners in the semantic aspects of vocabulary knowledge (see Chen \& Truscott, 2010; Heidari-Shahreza 
\& Tavakoli, 2012). Therefore, it is recommended that language teachers keep an eye to the L2 words which may lack an appropriate lexical match in the learners' L1. The teachers who come from the same linguistic background (as their learners do) can easily detect such troublesome words (NL words).

Within a broader scope, language teachers and materials writers should invest more time and energy on NL words. Extensive reading together with L2 marginal glosses using enhanced input may greatly help EFL learners to directly pick up the semantic features of such words, provided that it is placed within a systematic vocabulary recycling program (see Day \& Bamford, 2002; Heidari-Shahreza et al., 2014 a). Moreover, although L1-L2 translation is a popular (perhaps effective) strategy among EFL learners to deal with new L2 vocabulary (Tagashira, Kida \& Hoshino, 2010), due to the mismatch between L1 and L2 in case of NL words, it seems language teachers had better encourage their learners to learn these words directly from the contextual cues and not rely on their translations. Explicit vocabulary instruction and special strategy training, though, may be required to tackle NL words effectively (see Heidari-Shahreza, 2014; Heidari-Shahreza et al., 2014 a-f; Shintani, 2013 for some related studies).

\section{ACKNOWLEDHEMENTS}

The researchers would like to thank the participants of the study and the volunteers who kindly helped in the collection and validation of the target words.

\section{References}

[1] Barcroft J., Language Learning 52 (2002) 323-363.

[2] Barcroft J., Foreign Language Annals 37 (2004) 200-208.

[3] Chen C., Truscott J., Applied Linguistics 31 (2010) 693-713.

[4] Day R. R., Bamford J., Reading in a Foreign Language 14(2) (2002).

[5] Harding L., Language Testing 29 (2011) 163-180.

[6] Heidari-Shahreza M. A., Tavakoli M., The Language Learning Journal 40 (2012) 1-16.

[7] Heidari-Shahreza M. A., International Journal of Culture and History 1 (2014) 1-14.

[8] Heidari-Shahreza M. A., Moinzadeh A., Barati H. (a), GEMA Online Journal of Language Studies 14 (2014) 43-55.

[9] Heidari-Shahreza M. A., Moinzadeh A., Barati H. (b), International Journal of Research Studies in Language Learning 3 (2014) 83-96.

[10] Heidari-Shahreza M. A., Moinzadeh A., Barati H. (c), Modern Journal of Applied Linguistics 3 (2014) 81-91.

[11] Hemchua S., Schmitt N., Prospect 21 (2006) 3-25.

[12] Kirn E., Hartmann P. (2002). Interactions 1: Reading. New York: McGraw-Hill/ Contemporary.

[13] Nation I. S. P. (1990). Teaching and Learning Vocabulary. New York, NY: Heinle and Heinle Publishers. 
[14] Paribakht T. S., Language Learning 55 (2005) 701-748.

[15] Shintani N., TESOL Quarterly 47 (2013) 36-62.

[16] Sunderman G., Kroll J. F., Studies in Second Language Acquisition 28 (2006) 387-422.

[17] Tagashira K., Kida S., Hoshino Y., System 38 (2010) 412-421.

[18] Webb S., Applied Linguistics 28 (2007) 46-65.

[19] Mohammad Ali Heidari-Shahreza (d), International Letters of Social and Humanistic Sciences 20 (2014) 14-21.

[20] Mohammad Ali Heidari-Shahreza (e), International Letters of Social and Humanistic Sciences 25 (2014) 25-33.

[21] Mohammad Ali Heidari-Shahreza (f), International Letters of Social and Humanistic Sciences 2526 (2014) 88-96.

( Received 29 August 2014; accepted 08 September 2014)

\section{Appendix A. An example of the main reading passages}

- Bolded words represent lexicalized (L) words;

- Italic words represent non-lexicalized (NL) words.

He was a stubborn person and it explained why he had few friends. What was more, he was too abstinent; he had never gambled or even as an young adult, dated a girl. Yet, strangely enough, there was some rumor in the neighborhood that he used to smuggle cocaine and eloped with his girlfriend once to Las Vegas. As a retired artist, he most often sat in the lounge of Hotel Mirage, 3 minutes away from his home, to eat his brunch and giggle at the travelers who came and went. Some actually thought he was idiot. But indeed, he was just alone and tired of his whereabouts. Few people liked him. While he was real annoyance to the hotel staff, Mr. Hathaway, the hotel manager was kind to him because old George once had presented his masterpiece to the hotel. It had been hung on the wall at Mr. Hathaway's office for years. There was a joke among hotel staff about the artist. They said you could not flee from two persons: an undertaker and old George. Anyway, he suited the smoggy city of Chicago well; where your best friend is your pet, usually a dog and where you meet your neighbors accidentally at the junction of a road or on a wholesale market. 